\title{
Infant cardiopulmonary bypass: CD73 kinetics, association with clinical outcomes, and influence on serum adenosine production capacity
}

\author{
Jessica N. Persson ${ }^{1}$, Christine H. Baird ${ }^{1}$, Suhong Tong ${ }^{2}$, Tracy T. Urban ${ }^{3}$, Jelena Klawitter ${ }^{4}$, Paul E. Wischmeyer ${ }^{5}$ and
} Jesse A. Davidson ${ }^{1}$

BACKGROUND: Extracellular adenine nucleotides contribute to ischemia-reperfusion injury following infant cardiopulmonary bypass (CPB), whereas conversion to adenosine may be protective. Alkaline phosphatase (AP), a key enzyme responsible for this conversion, decreases after infant CPB. Indirect evidence suggests that soluble CD73 may simultaneously increase and partially offset this loss of AP. We sought to measure CD73 levels in infants undergoing CPB and determine its association with adenosine production capacity and postoperative support requirements.

METHODS: A prospective cohort study of infants $\leq 120$ days of age undergoing CPB. CD73 was measured before CPB and during rewarming. Multivariable modeling evaluated the contributions of CD73/AP to adenosine production capacity and postoperative support requirements.

RESULTS: Serum samples from 85 subjects were analyzed. The median CD73 concentration increased following CPB (95.2 vs. $179.8 \mathrm{ng} / \mathrm{ml} ; \quad P<0.0001)$. Rewarming CD73 was independently inversely associated with vasoactive inotropic support $(P<0.005)$ and length of intensive care unit stay $(P<0.005)$. Combined AP activity and CD73 concentration predicted adenosine production capacity $(P<0.0001)$.

CONCLUSIONS: Serum CD73 increases following infant CPB. Low rewarming CD73 is independently associated with increased postoperative support requirements. CD73 and AP together predict serum adenosine production capacity and may represent potential therapeutic targets to clear extracellular adenine nucleotides and improve outcomes following infant CPB.

A denosine triphosphate (ATP) is the main source of energy for biological cellular reactions. During times of inflammation, cell death, or ischemia and reperfusion, ATP is released from cells into the extracellular space, causing ongoing pathological inflammation. Extracellular ATP also stimulates vasoconstriction, activates platelets, and signals phagocytes to induce cell injury $(1,2)$.

ATP is broken down in a stepwise manner: first to adenosine diphosphate (ADP), then to adenosine monophosphate (AMP), and finally to adenosine. ADP and AMP are proinflammatory, functioning similarly to ATP. Adenosine, the final breakdown product of ATP, is protective against inflammation and ischemia-reperfusion injury (3). Adenosine accomplishes this via several mechanisms including vasodilation, platelet inhibition, inhibition of inflammatory mediators including platelet-activating factor, tumor necrosis factor alpha, and various chemokines (CCL3 and CCL4), and stimulation of Cox-2 activity to produce an anti-inflammatory prostaglandin (PGE2) $(2,4)$. Excessive neutrophil activation is also inhibited by adenosine, thereby reducing neutrophilinduced endothelial damage $(4,5)$. Preclinical studies have demonstrated that increased adenosine is associated with improved outcomes in animal models of sepsis, ischemiareperfusion, and proinflammatory states (6-8).

Enzymes responsible for this process of dephosphorylating ATP include alkaline phosphatase (AP) and soluble ectonucleotidases. These enzymes are of great interest, given their ability to clear extracellular ATP, increase favorable adenosine signaling, and potentially improve clinical outcomes (1,5,6,9-11). CD73, also known as ecto-5'-nucleotidase, is the rate-limiting enzyme responsible for converting extracellular AMP to adenosine (12). AP works in a similar manner to produce adenosine (1). Earlier in the dephosphorylation pathway, CD39, another ectonucleotide, functions to produce both ADP and AMP from ATP.

It has been hypothesized that extracellular adenine nucleotides may contribute to ischemia-reperfusion injury, vasoconstriction, and pathological inflammation observed following cardiac surgery with cardiopulmonary bypass (CPB) (3,13-15). Enzymes capable of dephosphorylating extracellular adenine nucleotides may in turn be protective. Our

${ }^{1}$ Department of Pediatrics, University of Colorado, Aurora, Colorado; ${ }^{2}$ Department of Biostatistics, University of Colorado, Aurora, Colorado; ${ }^{3}$ Research Institute, Children's Hospital Colorado/Department of Pediatrics, University of Colorado, Aurora, Colorado; ${ }^{4}$ Department of Anesthesiology, University of Colorado, Aurora, Colorado; ${ }^{5}$ Department of Anesthesiology and Surgery, Duke Clinical Research Institute, Duke University, Durham, North Carolina. Correspondence: Jessica N. Persson (jessica.persson@ childrenscolorado.org)

Received 31 August 2017; accepted 4 December 2017; advance online publication 17 January 2018. doi:10.1038/pr.2017.325 
group recently demonstrated a decrease in AP activity and a subsequent decrease in serum adenosine production capacity following CPB (16). Indirect evidence from our study suggests that soluble CD73 activity may increase after $\mathrm{CPB}$ and partially offset the observed loss of AP.

Currently, no study definitively describes the changes in CD73 levels in pediatric cardiology or following adult or pediatric cardiothoracic surgery. Therefore, we sought to understand the changes in soluble CD73 levels following infant $\mathrm{CPB}$ and its association with clinical outcomes. We hypothesized that CD73 levels would increase following CPB leading to an increase in AMP clearance capacity and be associated with a decrease in postoperative cardiovascular support, duration of mechanical ventilation, and length of intensive care unit stay.

\section{METHODS}

This study is a subanalysis of a larger prospective cohort examining $\mathrm{AP}$ in infants undergoing cardiothoracic surgery with $\mathrm{CPB}$ (17). The aims of the parent study were to (i) assess the kinetics of AP after infant CBP, (ii) measure the association between serum AP activity and clinical outcomes, as well as biomarkers of organ injury, and (iii) to evaluate the role of AP in determining serum capacity to clear AMP to adenosine. Patients were recruited and enrolled into the parent study at Children's Hospital Colorado between September 2013 and February 2016. All surgeries were performed at this institution, a tertiary care center with a dedicated pediatric cardiac intensive care unit (CICU) and cardiovascular operating rooms. Infants were enrolled in the parent study if they were $\leq 120$ days' old at the time of surgery and if $\mathrm{CPB}$ was used during surgery.

Exclusion criteria included corrected gestational age less than 34 weeks or weight less than $2 \mathrm{~kg}$ at the time of surgery. Subjects fulfilling these specific criteria were excluded because of the difference in AP activity in premature infants and the risk of anemia from research blood draws in lower-weight infants $(9,18)$. One hundred and twenty-two subjects were enrolled in the parent study. Subjects were then eligible for the substudy if there was adequate frozen serum to run CD73 analyses. Informed written parental consent was obtained for all study participants. The Colorado Multiple Institutional Review Board approved the protocol.

CPB was performed using a neonatal circuit consisting of a roller head pump (S5, LivaNova, Arvada, CO) and a Terumo FX05 oxygenator with a blood prime. The blood prime routinely underwent prebypass hemofiltration using a Minntech Hemocor HPH Junior hemoconcentrator (Medivators, Minneapolis, MN) with a polysulfone membrane before initiating bypass, allowing for partial filtration of molecules up to $65,000 \mathrm{Da}$. Anticoagulation was achieved before CPB by administering 500 units $/ \mathrm{kg}$ of heparin systemically to the patient. The initial target flow rate was $\sim 200 \mathrm{ml} / \mathrm{kg} / \mathrm{min}$. Cardioplegia was accomplished using del Nido formula cardioplegia solution at an initial dose of $30 \mathrm{ml} / \mathrm{kg}$ and subsequent dosing was considered after $60 \mathrm{~min}$ of aortic cross-clamp time.

The primary goal of the study was to measure the change in the CD73 level in infants undergoing $\mathrm{CPB}$ between induction of anesthesia and rewarming from bypass. Secondary objectives included measurement of CD73 levels through $24 \mathrm{~h}$ post operation, assessment of the association between CD73 levels and postoperative support, and establishing the relative associations of CD73 levels and AP activity with serum AMP clearance capacity.

Baseline and operative characteristics were acquired for all subjects. Serum samples were collected from indwelling venous or arterial catheters. These samples were drawn before CPB ("preoperative"), specifically between anesthesia induction and the first surgical incision, as well as during the rewarming phase of $\mathrm{CPB}$ ("rewarming"). Additional samples at 6- and 24-h postoperative time points for a subset of subjects were also available for analysis.

All samples were identically processed. A portion of the serum was used for the analysis of AP activity using a commercially available assay (Mayo Medical Laboratories, Rochester, MN). The remainder of the sample was frozen at $-70^{\circ} \mathrm{C}$ and stored for batch $\mathrm{CD} 73$ analysis. CD73 levels from each sample were measured using commercially available enzyme-linked immunosorbent assay kits specific for CD73 (MyBioSource, San Diego, CA). The assay was performed following the manufacturer's instructions. The clinical outcomes of interest were vasoactive inotropic score (VIS) at 6- and 24-h time points, CICU length of stay in days, length of time intubated in hours (19-21), and peak postoperative lactate. All outcomes were measured from the time of arrival to the CICU following completion of the surgery. Length of intubation was measured to the time of the first successful extubation. Peak lactate was assessed using lactate levels obtained as part of routine clinical care.

Conversion of 13C5-AMP to 13C5-adenosine was analyzed in a subset of serum samples using high-performance liquid chromatography-mass spectrometry (HPLC-MS) as previously published (16). Stored serum was assessed ex vivo via HPLC-MS/MS for conversion of exogenous 13C5-AMP to 13C5-adenosine (both from Toronto Research Chemicals, Toronto, ON, Canada). High concentration $(50 \mu \mathrm{mol} / \mathrm{l})$ of 13C5-AMP was used to simulate normal/ stress concentrations of AMP (11). Reactions were terminated after $15 \mathrm{~min}$ by addition of five volumes of acetonitrile/methanol containing $5 \mu \mathrm{mol} / \mathrm{l} \mathrm{d} 1$-adenosine as internal standard. In all assays, adenosine deaminase 1 (ADA1) and equilibrative nucleoside transporter 1 and 2 (ENT1,2) were blocked with erythro-9-(2hydroxy-3-nonyl)adenine (EHNA) and dipyridamole, respectively (Tocris Cookson; Bristol, UK), in order to prevent adenosine breakdown/reuptake, thus trapping the adenosine allowing accurate measurement of its production.

\section{Statistical Analysis}

Subjects' baseline characteristics were summarized using descriptive statistics both for the complete cohort and the predefined subgroups separated by the median CD73 level (upper vs. lower 50\%). All continuous variables that were not normally distributed were presented as median (range) in the tables. Wilcoxon rank-sum test was used to compare the medians. $\chi^{2}$-test was used to test the difference of proportions between groups. CD73 trend over time was analyzed in two ways. General linear mixed model was used to compare CD73 level change over time, with covariance structure that accounted for non-equally spaced time, whereas pairwise comparisons were conducted using $t$-test. Two-piecewise random coefficient mixed model was applied to compare the upward vs. downward slope of CD73 over time. Multivariable modeling was performed to assess the independent association of CD73 activity with clinical outcomes as well as serum adenosine production capacity. Initial potential covariates were selected based on clinical or biological significance. Spearman correlation test was performed to select candidate predictors for multivariable modeling. Covariates that were significantly associated with the outcome on univariate analysis were included in the initial model. Serum CD73 levels and AP activity were included as independent variables in all models based on a priori hypothesis. Distributions of continuous outcomes were inspected before modeling; natural log transformation was applied as indicated. General linear models were used for logtransformed outcomes and logistic regression models for dichotomized outcomes. Backward stepwise regression was used to determine the most parsimonious model. $P$ value $<0.05$ was considered statistically significant. SAS V9.4 (SAS Institute, Cary, NC) was used for data management and analyses; GraphPad Prism 6 was used for graphics. 


\section{Articles | Persson et al.}

\section{RESULTS}

A total of 85 subjects had both preoperative and rewarming samples available for $\mathrm{CD} 73$ measurement. These subjects were overall representative of the parent cohort. A detailed table of subject baseline and operative characteristics with comparison to the entire cohort is presented in Table $\mathbf{1}$.

The median CD73 levels roughly doubled (95.2-179.8 ng/ $\mathrm{ml}$ ) from the preoperative sample to the rewarming sample. This increase was statistically significant $(P$ value $<0.0001)$ with interquartile ranges of 55.5-154.9 and 128.9-293.3, respectively (Figure 1). An increase was seen in $94.1 \%$ of the subjects. Eighteen subjects also had 6- and 24-h postoperative samples available for analysis of $\mathrm{CD} 73$ levels. In these subjects, CD73 levels trended upward preoperatively to rewarming and then downtrended postoperatively with a peak at the rewarming time point. There was a statistically significant difference from the preoperative level to all postoperative time points $(P$ value $<0.0001)$. In addition, there was a statistically significant difference from rewarming level and 24-h postoperative level, but not the 6-h postoperative level ( $P$ values $=0.029$ and 0.612 , respectively). Finally, the preoperative-to-rewarming slope was significantly different from the rewarming-to-24-h-postoperative slope $(P$ value $=0.0009$ ), indicating a rapid rise and relatively slow decline in CD73 levels. The trend for these 18 subjects is presented in Figure 2.

We next divided subjects into upper 50th\% vs. lower 50th\% CD73 levels to analyze whether elevated CD73 was associated with specific baseline and operative clinical characteristics. Subjects with higher preoperative CD73 levels demonstrated a significantly higher rate of preoperative mechanical ventilation and inotropic support compared with their counterparts with lower preoperative CD73 levels $(P$ value $=0.0002$ and 0.014 , respectively; Table 2). Conversely, subjects with lower rewarming CD73 levels were younger $(P$ value $=0.007)$, more often displayed single-ventricle physiology $(P$ value $=0.008)$, and more often underwent selective cerebral perfusion $(P$ value $=0.006$; Table 3 ).

On univariate analysis of clinical outcomes, subjects with lower rewarming CD73 levels had significantly higher VIS at $24 \mathrm{~h}$ with a mean of $10.3(\mathrm{SD} \pm 5.6)$ vs. $7.3(\mathrm{SD} \pm 4.5 ; P$ value $=0.008$ ) and longer CICU length of stay with a median of 7 days (interquartile range (IQR) $4.5-17$ ) vs. 4 days (IQR 3-7; $P$ value $=0.010)$. Similar trends were seen for VIS at $6 \mathrm{~h}$ with a mean of $10.6(\mathrm{SD} \pm 4.4)$ vs. $9.5(\mathrm{SD} \pm 4.0 ; P$ value $=$ 0.224 ) and length of mechanical ventilation with a median of $50.9 \mathrm{~h}$ (IQR 24.7-93.4) vs. $35.8 \mathrm{~h}$ (IQR 20.1-67.8; $P$ value $=$ 0.057); however, these did not reach statistical significance.

We then assessed whether CD73 maintained an independent association with VIS at $24 \mathrm{~h}$ and CICU length of stay when controlling for key operative factors as well as perioperative AP activity. Multivariable modeling results are depicted in Tables $\mathbf{4}$ and $\mathbf{5}$ and the full models are shared in the Supplementary Tables S1a-S1e online). The rewarming CD73 level demonstrated an independent inverse association with VIS at $24 \mathrm{~h}(P$ value $=0.004)$ and CICU
Table 1. Baseline and operative characteristics

\begin{tabular}{|c|c|c|c|}
\hline Characteristics & $\begin{array}{l}\text { CD73 study } \\
(N=85)\end{array}$ & $\begin{array}{l}\text { Full cohort } \\
(N=122)\end{array}$ & $P$ value \\
\hline Gender & & & 1.000 \\
\hline Male & $57 \%(n=48)$ & $55.7 \%(n=68)$ & \\
\hline Age at surgery (days) & $13(2-120)$ & $15(1-120)$ & 0.901 \\
\hline Weight (kg) & $3.5(2.1-7.9)$ & $3.5(2.1-7.9)$ & 0.784 \\
\hline Ethnicity & & & 0.865 \\
\hline White & $53 \%(n=45)$ & $56.2 \%(n=68)$ & \\
\hline Hispanic & $36 \%(n=30)$ & $32 \%(n=39)$ & \\
\hline Other & $11 \%(n=9)$ & $12 \%(n=14)$ & \\
\hline $\begin{array}{l}\text { Aristotle score- } \\
\text { comprehensive }\end{array}$ & $9.5(3-16)$ & $9(3-15)$ & 0.841 \\
\hline Single-ventricle physiology & $\begin{array}{l}32.1 \% \\
(n=27)\end{array}$ & $32 \%(n=38)$ & 1.000 \\
\hline Preoperative intubation & $35 \%(n=30)$ & $32 \%(n=39)$ & 0.647 \\
\hline $\begin{array}{l}\text { Preoperative inotropic } \\
\text { support }\end{array}$ & $\begin{array}{l}14.1 \% \\
(n=12)\end{array}$ & $12.3 \%(n=15)$ & 0.702 \\
\hline Preoperative steroid & $43 \%(n=36)$ & $44 \%(n=53)$ & 0.853 \\
\hline $\begin{array}{l}\text { Cardiopulmonary bypass time } \\
\text { (min) }\end{array}$ & $144(54-399)$ & $137(0-399)$ & 0.414 \\
\hline Cross-clamp time (min) & $76(0-241)$ & $74(0-241)$ & 0.394 \\
\hline $\begin{array}{l}\text { Deep hypothermic circulatory } \\
\text { arrest (min) }\end{array}$ & $0(0-154)$ & $0(0-154)$ & 0.959 \\
\hline $\begin{array}{l}\text { Selective cerebral perfusion } \\
\text { (min) }\end{array}$ & $0(0-82)$ & $0(0-115)$ & 0.307 \\
\hline
\end{tabular}

Continuous data are expressed as the median (range) and categorical data are expressed as $n$ (\%).

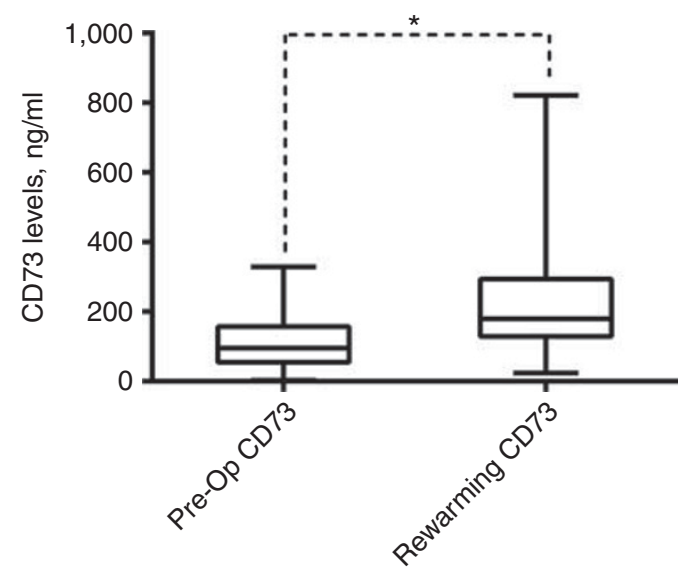

Figure 1. The median $\mathrm{CD} 73$ level before $\mathrm{CPB}$ and during rewarming; ${ }^{*} P<0.0001$. CPB, cardiopulmonary bypass.

length of stay $(P$ value $=0.002)$. Interestingly, for a fixed rewarming CD73 level, higher preoperative CD73 level was associated with an increased postoperative support, suggesting that both relative and absolute increase in CD73 may be clinically important. 


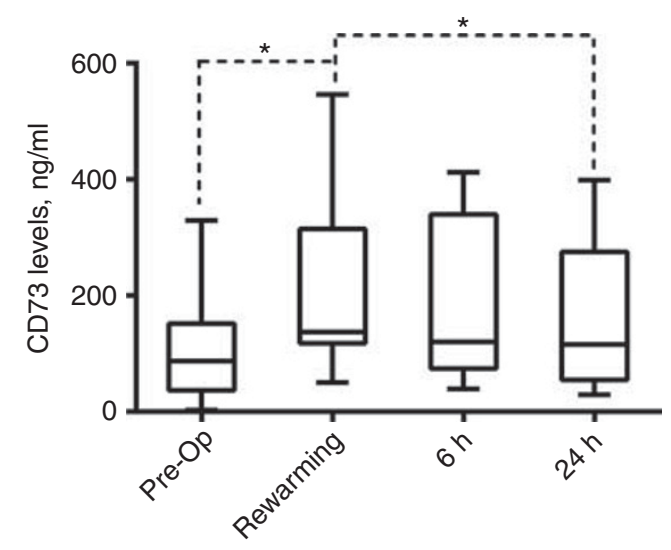

Figure 2. Trend of the median CD73 level over time in subjects with CD73 levels at four time points $(n=18) ;{ }^{*} P<0.05$.

Table 2. Preoperative subject characteristics and preoperative CD73 levels

\begin{tabular}{|c|c|c|c|}
\hline \multirow[t]{2}{*}{ Preoperative characteristics } & \multicolumn{2}{|c|}{ Preoperative CD73 level } & \multirow[t]{2}{*}{$P$ value } \\
\hline & $\begin{array}{l}<50 \% \\
n=43\end{array}$ & $\begin{array}{l}\geq 50 \% \\
n=42\end{array}$ & \\
\hline \multicolumn{4}{|l|}{ Gender } \\
\hline Male & $24(55.8 \%)$ & $24(57.1 \%)$ & 0.902 \\
\hline Age, days & $8(2,120)$ & $14(2,109)$ & 0.782 \\
\hline Weight, kg & $3.8(2.6-7.4)$ & $3.3(2.1-7.9)$ & 0.141 \\
\hline Ethnicity & & & 0.9580 \\
\hline White & $23(53.5 \%)$ & $22(53.7 \%)$ & \\
\hline Hispanic & $15(34.9 \%)$ & $15(36.6 \%)$ & \\
\hline Other & $5(11.6 \%)$ & $4(9.8 \%)$ & \\
\hline $\begin{array}{l}\text { Aristotle score-- } \\
\text { comprehensive }\end{array}$ & $9(3,14.5)$ & $10(3,16)$ & 0.770 \\
\hline Single-ventricle physiology & $15(34.9 \%)$ & $12(29.3 \%)$ & 0.582 \\
\hline $\begin{array}{l}\text { Preoperative mechanical } \\
\text { ventilation }\end{array}$ & $7(16.3 \%)$ & $23(54.8 \%)$ & 0.0002 \\
\hline Preoperative inotropic support & $2(4.7 \%)$ & $10(23.8 \%)$ & 0.014 \\
\hline Preoperative steroid use & $22(52.4 \%)$ & $26(61.9 \%)$ & 0.378 \\
\hline
\end{tabular}

Following identification of this independent inverse association of rewarming CD73 with vasoactive and inotropic support, we wanted to assess whether CD73 was also associated with biochemical evidence of poor postoperative oxygen delivery. We, therefore, performed an additional limited post hoc analysis of rewarming CD73 levels and peak postoperative lactate. Rewarming CD73 levels demonstrated an independent inverse association with peak lactate (decrease in lactate of $0.5 \mathrm{mmol} / \mathrm{l}$ for every $100 \mathrm{ng} / \mathrm{ml}$ increase in CD73;
Table 3. Baseline and operative subject characteristics and rewarming CD73 levels

\begin{tabular}{|c|c|c|c|}
\hline \multirow[t]{2}{*}{ Subject characteristics } & \multicolumn{2}{|c|}{ Rewarming CD73 level } & \multirow[t]{2}{*}{$P$ value } \\
\hline & $\begin{array}{l}<50 \% \\
n=43\end{array}$ & $\begin{array}{l}\geq 50 \% \\
n=43\end{array}$ & \\
\hline \multicolumn{4}{|l|}{ Gender } \\
\hline Male & $26(60.5 \%)$ & $22(51.2 \%)$ & 0.385 \\
\hline Age, days & $6(2,120)$ & $32(4,118)$ & 0.007 \\
\hline Weight, kg & $3.3(2.1,7.4)$ & $3.6(2.1,7.9)$ & 0.412 \\
\hline Ethnicity & & & 0.1658 \\
\hline White & $20(46.5 \%)$ & $26(61.9 \%)$ & \\
\hline Hispanic & $16(37.2 \%)$ & $14(33.3 \%)$ & \\
\hline Other & $7(16.3 \%)$ & $2(4.8 \%)$ & \\
\hline $\begin{array}{l}\text { Aristotle score-comprehensive } \\
\text { (median (interquartile range)) }\end{array}$ & $10(8,14.5)$ & $9(6.3,11)$ & 0.080 \\
\hline Single-ventricle physiology & $19(45 \%)$ & $8(18.6 \%)$ & 0.008 \\
\hline $\begin{array}{l}\text { Preoperative mechanical } \\
\text { ventilation }\end{array}$ & $13(30 \%)$ & 17 (39.5\%) & 0.366 \\
\hline Preoperative inotropic support & $5(11.6 \%)$ & $7(16.3 \%)$ & 0.534 \\
\hline Preoperative steroid & $28(65.1 \%)$ & $21(50 \%)$ & 0.159 \\
\hline $\begin{array}{l}\text { CPB time, minutes (median } \\
\text { (interquartile range)) }\end{array}$ & $\begin{array}{l}154(116 \\
201)\end{array}$ & $\begin{array}{l}122(88, \\
204)\end{array}$ & 0.165 \\
\hline Cross-clamp time, min & $79(68,96)$ & $74(49,115)$ & 0.392 \\
\hline $\begin{array}{l}\text { Deep hypothermic circulatory } \\
\text { arrest, min }\end{array}$ & $2(0,10)$ & $0(0,6)$ & 0.135 \\
\hline Selective cerebral perfusion, min & $0(0,46)$ & $0(0,0)$ & 0.006 \\
\hline
\end{tabular}

Table 4. Multivariable modeling of CD73 with VIS at $24 \mathrm{hVIS}$, vasoactive inotropic score.

\begin{tabular}{lcc}
\hline $\begin{array}{l}\text { Independent } \\
\text { variable }\end{array}$ & $\begin{array}{c}\text { Change in 24-h VIS per 10-unit } \\
\text { increase in independent variable (\%) }\end{array}$ & $P$ value \\
\hline Preoperative CD73 & 2 & 0.024 \\
Rewarming CD73 & -2 & 0.004 \\
\hline
\end{tabular}

$P$ value $=0.01)$, but only when adjusted for CD73 levels at later time points $(6$ or $24 \mathrm{~h})$, where persistent elevation was associated with higher peak lactate $(P$ value $=0.002)$, VIS at $24 \mathrm{~h}(P$ value $=0.0004)$, and CICU length of stay $(P$ value $=0.004)$.

The final portion of this study sought to determine whether the combined measurement of soluble CD73 and AP activity predicted serum adenosine production capacity. Adenosine production capacity was measured in 21 of the rewarming study samples. The combined rewarming AP activity and CD73 level significantly predicted serum ability to convert AMP to adenosine $\left(R^{2}=0.52\right.$; Table 6). Overall, AP 
Table 5. Multivariable modeling of CD73 with ICU length of stay.

\begin{tabular}{lcc}
\hline Independent variable & $\begin{array}{c}\text { Change in ICU length of } \\
\text { stay per 10-unit increase in } \\
\text { independent variable (\%) }\end{array}$ & $P$ value \\
\hline Preoperative CD73 & 5 & 0.006 \\
Rewarming CD73 & -6 & 0.002 \\
\hline
\end{tabular}

ICU, intensive care unit.

Table 6. Multivariable analysis of $\mathrm{CD} 73, \mathrm{AP}$, and ex vivo adenosine production capacity.

\begin{tabular}{lcc}
\hline $\begin{array}{l}\text { Independent } \\
\text { variable }\end{array}$ & $\begin{array}{c}\text { Change in adenosine per 10-unit } \\
\text { increase in independent variable (\%) }\end{array}$ & $P$ value \\
\hline Rewarming CD73 & 10 & 0.895 \\
Rewarming AP & 70 & $<0.0001$ \\
\hline AP, alkaline phosphatase. & &
\end{tabular}

contributed substantially more to the model than CD73 ( $P$ value $<0.0001)$, consistent with our ex vivo studies of the effects of AP blockade (16).

\section{DISCUSSION}

In this study, we present for the first time the novel finding of a consistent and significant rise in serum CD73 levels stimulated by infant cardiac surgery with $\mathrm{CPB}$. Soluble CD73 and AP explain a substantial portion of the variability in serum adenosine production capacity, although CD73 plays a much smaller role than AP. Despite the relatively small contribution to serum adenosine production capacity, however, CD73 is independently associated with 24-h VIS and CICU length of stay even after controlling for AP.

Our finding of increased soluble CD73 following infant CPB is consistent with preclinical and adult clinical studies of hypoxia and ischemia-reperfusion injury. CPB causes hypoxia, inflammation, ischemia-reperfusion injury, and subsequent organ dysfunction (22-24). These same factors are responsible for triggering expression of CD73 through several mechanisms in a variety of tissues. Hypoxia-inducible transcription factor $(\mathrm{HIF}-1 \alpha)$ is the key molecule responsible for upregulating CD73 (10,25). Kobayashi et al. demonstrated an increased expression of CD73 in rat pheochromocytoma cells exposed to chronic hypoxia (26). Furthermore, CD73 expression via the HIF-1 $\alpha$ induction pathway was increased in liver samples of adults suffering from acute liver failure, as well as in renal tissue of mice following ischemic preconditioning (short periods of renal artery occlusion) $(27,28)$. Similarly, Kim et al. suggested expression of CD73 via TGF- $\beta 1$ induction in renal tissue of mice that underwent anesthesia with isoflurane, a known renal TGF- $\beta 1$ stimulator (29). Interferon-beta, an immunomodulatory cytokine, has also been shown to upregulate CD73 expression on endothelial cells $(30,31)$.

Although the immediate effects of hypoxic or inflammatory insults on CD73 expression are well established in non-CPB pathology, to our knowledge, the change in the soluble CD73 level over time has not been clearly defined in any human disease. Ramakers et al. demonstrated that CD73 expression initially increased following administration of endotoxin in the serum of volunteer adults but was markedly reduced within $24 \mathrm{~h}$ (ref. 32). Our study also noted a slow decrease in soluble CD73 levels through $24 \mathrm{~h}$ postoperatively in a small subset of the cohort with 6- and 24-h serum samples available for analysis. Although our preliminary data are consistent with the finding of Ramakers et al., further examination of CD73 kinetics and potential associated clinical factors is warranted.

When applied clinically, pre- and postoperative CD73 levels demonstrated opposite associations with the condition of our subjects. On average, sicker subjects had higher preoperative CD73 levels but relatively lower postoperative CD73 levels on univariate analysis. These findings were maintained on multivariable analysis, where higher preoperative CD73 levels but comparatively lower postoperative levels were independently associated with increased postoperative support requirements. Cautious interpretation of a limited number of subjects with CD73 levels available from later time points suggests an association of persistent CD73 elevation with higher peak lactate levels, VIS, and CICU length of stay. Although these findings at first appear contradictory, they are consistent with the current understanding of CD73 expression and physiological effects: an ischemic and/or hypoxic insult is necessary to drive increased expression, a more robust initial CD73 response may be protective, but persistence of the insult may result in a prolonged stimulus for $\mathrm{CD} 73$ expression $(10,25,33,34)$. Eckle et al. demonstrated an induction of CD73 in the lung with mechanical ventilation (7). This increase in CD73 may help offset negative effects of mechanical ventilation including ventilator-induced lung injury and breakdown of the pulmonary capillary-alveolar barrier (7). Further research into the specific drivers of CD73 expression following congenital heart surgery is required.

The potentially protective role of CD73 derives from its ability to dephosphorylate AMP to adenosine. Adenosine, as previously described, works to reduce inflammation and decrease damage associated with ischemic insults through multiple mechanisms (35). Adenosine stimulates vasodilation by enhancing arterial endothelial cell production of nitric oxide, and increased CD73-derived adenosine production may promote blood flow to improve oxygenation to tissues during times of hypoxia $(10,36)$. Platelet aggregation is inhibited by adenosine, via adenylate cyclase action on endothelial cell A2a receptors, which may help avoid microvascular thrombus formation (4). Adenosine also prevents inflammatory tissue injury through reduction of vascular leakage and binding to neutrophil receptors to inhibit their accumulation $(5,10,37)$. However, few data exist to demonstrate the role of $\mathrm{CD} 73$ in cardiovascular disease. Koszalka et al. reported CD73 to be the major enzyme stimulating adenosine formation in cardiac tissues and large blood vessel endothelium of mice (38). Extracellular CD73 may also be cardioprotective, preventing vascular leakage caused by hypoxia $(7,39)$. 
Pettengill et al. recently demonstrated that soluble CD73 and AP determine serum capacity to convert labeled AMP to adenosine in ex vivo samples from healthy neonates (11). Our group subsequently identified a strong association between AP and serum capacity to dephosphorylate AMP to adenosine after infant CBP (16). In this cohort, serum adenosine production capacity consistently decreased from preoperation to rewarming and was tightly correlated with a simultaneous drop in serum AP activity. In subjects with only a small decrease in AP activity, however, serum capacity to produce adenosine from AMP actually increased between preoperation and rewarming. Importantly, this increase was abolished with CD73 inhibition, suggesting that CD73 levels likely rise after surgery in this population and can partially offset the loss of serum AP activity.

Our current findings confirm that CD73 levels increase after infant CBP. In addition, as expected from our prior studies, postoperative CD73 levels and AP activity together predict the capacity to produce serum adenosine; however, serum AP contributes substantially more to this process than CD73. As the change in AP reaches a certain level, it likely overwhelms CD73's contribution to serum adenosine production. The surprising result of our current study, though, is the substantial independent association of $\mathrm{CD} 73$ with postoperative support requirements despite its relatively small contribution to serum adenosine production capacity. Several possibilities exist to explain this discrepancy. The first is that failure to increase CD73 is simply a marker of sicker patients and the association with postoperative support is confounded by changes in other molecular pathways. A second possibility is that CD73 has alternative protective actions beyond conversion of AMP to adenosine. Perhaps, the most likely etiology, however, is the role of tissue-based CD73, which we did not examine in this study. CD73 can be soluble in the serum or bound to the surface of epithelial and endothelial cells, and, as a result, is found throughout the body on vascular endothelium and in a multitude of organs including the intestines, kidney, liver, and heart $(10,12,40)$. Although our study found an increase in soluble CD73 after CPB, we did not measure the effect of CPB on tissue-based CD73. Tissue-based CD73 has been shown to decrease epithelial and endothelial permeability on various tissues, which likely plays an important role following infant CPB (10). Further studies are needed to characterize these changes in tissue CD73 expression after CPB.

Looking forward, it is possible that either CD73, AP, or both could serve as potential therapeutic targets to clear extracellular adenine nucleotides and improve clinical outcomes in infants following CPB. Although several small studies demonstrate the effects of supplemental AP, the role of CD73 supplementation is less understood (41-44). Hart et al. demonstrated that soluble CD73 supplementation attenuated PMN infiltration, thereby leading to significantly lower levels of intestinal injury in CD73-negative mice with intestinal ischemia-reperfusion injury (38). CD73 administered to mechanically ventilated mice led to reduced lung tissue damage and increased adenosine levels in the lung (7). Similarly, Grenz et al. documented decreased renal injury in mice undergoing acute renal hypoxia after receiving CD73 (ref. 28). In patients with ARDS, Bellingan et al. found reduced mortality when given interferon-beta-1a as it was thought to upregulate CD73 expression in the lungs (30). These discoveries suggest a possible role for CD73 therapy to reduce inflammation and ischemia-reperfusion injury. However, further research is necessary to assess the effects of CD73 supplementation in humans.

Several limitations existed in this study. First, the primary cohort was not designed to assess CD73. As a result, there were a limited number of subjects with sufficient serum samples available to measure CD73 and serum adenosine production capacity. Similarly, only a subset of subjects had samples obtained at the 6- and 24-h postoperative time points, leading to small sample sizes and limited statistical analysis at 6 and $24 \mathrm{~h}$ post operation, particularly for multivariable modeling of CD73 and peak lactate. The blood volumes available for sampling varied because of what was clinically allowable for every subject at each given time point. From a mechanistic perspective, an ex vivo assessment of adenosine production, through AMP clearance capacity, was used as a surrogate to reflect adenosine levels, as rapid cellular uptake of adenosine makes serum measurements difficult to assess outside of the steady state. As previously discussed, only serum levels of CD73 were analyzed in our study. The role of cellular-based CD73 on inflammation and outcomes following $\mathrm{CPB}$ requires further study to better understand the clinical impact of perioperative CD73. On a similar note, larger analysis of $\mathrm{CD} 73$ in conjunction with $\mathrm{AP}$ is necessary to better understand the independent impact of CD73. Finally, as with all observational studies, we can only assess association rather than causation. Further animal models and ultimately clinical trials are necessary to fully determine the role of CD73 in host protection after CBP and if routine monitoring would be beneficial.

\section{CONCLUSIONS}

The serum CD73 level consistently increases in infants following $\mathrm{CPB}$ and starts decreasing by $24 \mathrm{~h}$. Higher preoperative CD73 levels and lower rewarming CD73 levels are independently associated with an increased vasoactive inotropic score at $24 \mathrm{~h}$ post operation and length of intensive care unit stay. CD73 predicts serum adenosine production capacity when assessed in conjunction with AP. Further study is warranted to explore the role of tissue-based CD73 after infant $\mathrm{CBP}$, as well as to assess potential CD73 monitoring or therapeutic administration in this population.

\section{SUPPLEMENTARY MATERIAL}

Supplementary material is linked to the online version of the paper at http://www.nature.com/pr

\section{ACKNOWLEDGMENTS}

We recognize the nurses and staff of the CICU and CTRC at Children's Hospital Colorado for their valuable contributions to our project. 


\section{STATEMENT OF FINANCIAL SUPPORT}

Davidson and this study are supported by NIH/NCATS Colorado CTSA Grant Number UL1 TR001082, AHA 13CRP17300016, and NIH/NHLBI 1K23HL123634-01.

\section{DISCLAIMER}

The contents are the authors' sole responsibility and do not necessarily represent official NIH views.

Disclosure: The authors declare no conflict of interest.

\section{REFERENCES}

1. Eltzschig HK, Sitkovsky MV, Robson SC. Purinergic signaling during inflammation. N Engl J Med 2012;367:2322-33.

2. Bours MJL, Swennen ELR, Virgilio FD, et al. Adenosine 5'-triphosphate and adenosine as endogenous signaling molecules in immunity and inflammation. Pharmacol Ther 2006;112:358-404.

3. Eltzschig HK, Eckle T. Ischemia and reperfusion - from mechanism to translation. Nat Med 2001;17:1391-401.

4. Kawashima Y, Nagasawa T, Ninomiya H. Contribution of ecto-5'nucleotidase to the inhibition of platelet aggregation by human endothelial cells. Blood 2000;96:2157-62.

5. Eltzschig HK, Thompson LF, Karhausen J, et al. Endogenous adenosine produced during hypoxia attenuates neutrophil accumulation: coordination by extracellular nucleotide metabolism. Blood 2004;104: 3986-92.

6. Hasko G, Csoka B, Koscso B, et al. Ecto-5'-Nucleotidase (CD73) decreases mortality and organ injury in sepsis. J Immunol 2011;187: 4256-67.

7. Eckle T, Fullbier L, Wehrmann $M$, et al. Identification of ectonucleotidases CD39 and CD73 in innate protection during acute lung injury. J Immunol 2007;178:8127-36.

8. Louis NA, Robinson AM, MacManus CF, et al. Control of IFN-alphaA by CD73: implications for mucosal inflammation. J Immunol 2008;180: 4246-55.

9. Davidson J, Tong S, Hauck A, et al. Alkaline phosphatase activity after cardiothoracic surgery in infants and correlation with post-operative support and inflammation: a prospective cohort study. Crit Care 2012;16: R160.

10. Colgan SP, Eltzschig HK, Eckle T, et al. Physiologic roles for ecto-5'nucleotidase (CD73). Purinergic Signal 2006;2:351-60.

11. Pettengill M, Robson S, Tresenriter M, et al. Soluble ecto-5'-nucleotidase ( $5^{\prime} \mathrm{NT}$ ), alkaline phosphatase, and adenosine deaminase (ADA1) activities in neonatal blood favor elevated extracellular adenosine. J Biol Chem 2013;288:27315-6.

12. Zimmermann $H$, Zebisch $M$, Strater $N$. Cellular function and molecular structure of ecto-nucleotidases. Purinergic Signal 2012;8: 437-502.

13. Hoegl S, Zwissler B, Eltzschig HK, et al. Acute respiratory distress syndrome following cardiovascular surgery: current concepts and novel therapeutic approaches. Curr Opin Anaesthesiol 2016;29: 94-100.

14. Lu D, Insel PA. Cellular mechanisms of tissue fibrosis. 6. Purinergic signaling and response in fibroblasts and tissue fibrosis. Am J Physiol Cell Physiol 2013;206:C779-88.

15. Hui Y, Zhao SS, Love JA, et al. Development and application of a LC-MS/ MS method to quantify basal adenosine concentration in human plasma from patients undergoing on-pump CABG surgery. J Chromatogr B Analyt Technol Biomed Life Sci 2012;885-886:30-6.

16. Davidson JA, Urban T, Tong S, et al. Alkaline phosphatase, soluble extracellular adenine nucleotides, and adenosine production after infant cardiopulmonary bypass. PLoS ONE 2016;11:e0158981.

17. Davidson JA, Urban T, Baird C, et al. Alkaline phosphatase in infant cardiopulmonary bypass: kinetics and relationship to organ injury and major cardiovascular events. J Peds 2017;190:49-54.
18. Crofton PM, Hume R. Alkaline phosphatase isoenzymes in the plasma of preterm and term infants: serial measurements and clinical correlations. Clin Chem 1987;33:1783-7.

19. Gaies MG, Gurney JG, Yen AH, et al. Vasoactive-inotropic score as a predictor of morbidity and mortality in infants after cardiopulmonary bypass. Pediatr Crit Care Med 2010;11:234-8.

20. Davidson J, Tong S, Hancock $H$, et al. Prospective validation of the vasoactive-inotropic score and correlation to short-term outcomes in neonates and infants after cardiothoracic surgery. Intensive Care Med 2012;38:1184-90.

21. McIntosh AM, Tong S, Deakyne SJ, et al. Validation of the vasoactiveinotropic score in pediatric sepsis. Pediatr Crit Care Med 2017;18:750-7.

22. Raja SG, Dreyfus GD. Modulation of systemic inflammatory response after cardiac surgery. Asian Cardiovasc Thorac Ann 2005;13:382-95.

23. Klein DJ, Briet F, Nisenbaum R, et al. Endotoxemia related to cardiopulmonary bypass is associated with increased risk of infection after cardiac surgery: a prospective observational study. Crit Care 2011;15:R69.

24. Ascione R, Talpahewa S, Rajakaruna C, et al. Splanchnic organ injury during coronary surgery with or without cardiopulmonary bypass: a randomized, controlled trial. Ann Thorac Surg 2006;81:97-103.

25. Synnestvedt K, Furuta GT, Comerford KM, et al. Ecto-5'-nucleotidase (CD73) regulation by hypoxia-inducible factor-1 mediates permeability changes in intestinal epithelia. J Clin Invest 2002;110:993-1002.

26. Kobayashi S, Zimmermann H, Millhorn DE. Chronic hypoxia enhances adenosine release in rat $\mathrm{PC} 12$ cells by altering adenosine metabolism and membrane transport. J Neurochem 2000;74:621-32.

27. Tak E, Jung DH, Kim SH, et al. Protective role of hypoxia-inducible factor-1alpha-dependent CD39 and CD73 in fulminant liver failure. Toxicol Appl Pharmacol 2017;314:72-81.

28. Grenz A, Zhang H, Eckle T, et al. Protective role of ecto-5'-nucleotidase (CD73) in renal ischemia. J Am Soc Nephrol 2007;18:833-45.

29. Kim M, Ham A, Kim JY, et al. The volatile anesthetic isoflurane induces ecto-5'-nucleotidase $(\mathrm{CD} 73)$ to protect against renal ischemia and reperfusion injury. Kidney Int 2013;84:90-103.

30. Belligan G, Maksimow M, Howell DC, et al. The effect of intravenous interferon-beta-1a (FP-1201) on lung CD73 expression and on acute respiratory distress syndrome mortality: an open-label study. Lancet Respir Med 2014;2:98-107.

31. Kiss J, Yegutkin GG, Koskinen K, et al. IFN- $\beta$ protects from vascular leakage via up-regulation of CD73. Eur J Immunol 2007;37:3334-8.

32. Ramakers BP, Wever KE, Kox M, et al. How systemic inflammation modulates adenosine metabolism and adenosine receptor expression in humans in vivo. Crit Care Med 2012;40:2609-16.

33. Zhou Y, Murthy JN, Zeng D, et al. Alterations in adenosine metabolism and signaling in patients with chronic obstructive pulmonary disease and idiopathic pulmonary fibrosis. PLoS ONE 2010;5:e9224.

34. Qing M, Schumacher K, Heise R, et al. Intramyocardial synthesis of proand anti-inflammatory cytokines in infants with congenital cardiac defects. JACC 2003;41:2266-74.

35. Antonioli L, Pacher P, Vizi ES, et al. CD39 and CD73 in immunity and inflammation. Trends Mol Med 2013;19:355-67.

36. Li JM, Fenton RA, Cutler BS, et al. Adenosine enhances nitric oxide production by vascular endothelial cells. Am J Physiol 1995;269:C519-23.

37. Hart ML, Henn M, Köhler D, et al. Role of extracellular nucleotide phosphohydrolysis in intestinal ischemia-reperfusion injury. FASEB J 2008;22:2784-97.

38. Koszalka P, Ozuyama B, Huo Y, et al. Targeted disruption of cd73/Ecto5 '-nucleotidase alters thromboregulation and augments vascular inflammatory response. Circ Res 2004;95:814-21.

39. Thompson LF, Eltzschig HK, Ibla JC, et al. Crucial role for ecto-5'nucleotidase $(\mathrm{CD} 73)$ in vascular leak during hypoxia. J Exp Med 2004;200:1395-405.

40. Eltzschig HK, Ibla JC, Furuta GT, et al. Coordinated adenine nucleotide phosphohydrolysis nucleoside signaling in posthypoxic endothelium: role of ectonucleotidases and adenosine $A_{2 B}$ receptors. J Exp Med 2003;198: 783-96. 
41. Heemskerk S, Masereeuw R, Moesker O, et al. Alkaline phosphatase treatment improves renal function in severe sepsis or septic shock patients. Crit Care Med 2009;37:417-23, e1.

42. Pickkers P, Heemskerk S, Schouten J, et al. Alkaline phosphatase for treatment of sepsis-induced acute kidney injury: a prospective randomized double-blind placebo-controlled trial. Crit Care 2012; 16:R14.

43. Kats S, Brands R, Seinen W, et al. Anti- inflammatory effects of alkaline phosphatase in coronary artery bypass surgery with cardiopulmonary bypass. Recent Pat Inflamm Allergy Drug Discov 2009;3: 214-0.

44. Kats S, Schonberger JP, Brands R, et al. Endotoxin release in cardiac surgery with cardiopulmonary bypass: pathophysiology and possible therapeutic strategies. An update. Eur J Cardiothorac Surg 2011;39:451-8.
This work is licensed under a Creative Commons Attribution-NonCommercial-NoDerivs

4.0 International License. The images or other third party material in this article are included in the article's Creative Commons license, unless indicated otherwise in the credit line; if the material is not included under the Creative Commons license, users will need to obtain permission from the license holder to reproduce the material. To view a copy of this license, visit http://creativecommons.org/licenses/ by-nc-nd/4.0/

(c) The Author(s) (2018) 